\title{
On the existence of two states in liquid water: Impact on biological and nanoscopic systems
}

Article in International Journal of Nanotechnology · January 2016

DOI: $10.1504 /$ IJNT.2016.079670

CITATIONS

0

10 authors, including:

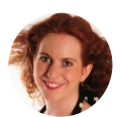

Laura Martinez Maestro

University of Oxford

32 PUBLICATIONS 1,536 CITATIONS

SEE PROFILE

\section{Francesco Mallamace}

Massachusetts Institute of Technology

210 PUBLICATIONS 3,986 CITATIONS

SEE PROFILE

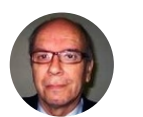

Francisco Jaque

Universidad Autónoma de Madrid

236 PUBLICATIONS 2,193 CITATIONS

SEE PROFILE

H. Eugene Stanley

Boston University

1,630 PUBLICATIONS 104,150 CITATIONS

SEE PROFILE

Some of the authors of this publication are also working on these related projects: 


\title{
On the existence of two states in liquid water: impact on biological and nanoscopic systems
}

\author{
L.M. Maestro \\ Clarendon Laboratory, \\ Department of Physics, \\ University of Oxford, \\ Parks Road, Oxford OX1 3PU, UK \\ Email: laura.martinezmaestro@physics.ox.ac.uk
}

\section{M.I. Marqués*}

Departamento de Física de Materiales, Universidad Autónoma de Madrid, Madrid, 28049, Spain

and

Condensed Matter Physics Center (IFIMAC)

and Instituto Nicolás Cabrera,

Universidad Autónoma de Madrid,

Madrid, 28049, Spain

Email: manuel.marques@uam.es

${ }^{*}$ Corresponding author

\section{E. Camarillo}

Institute of Physics,

UNAM,

Mexico DF, 04510, México

Email: cgarcia@fisica.unam.mx

\section{Jaque and J. García Solé}

Departamento de Física de Materiales, Universidad Autónoma de Madrid,

Madrid, 28049, Spain

Email: daniel.jaque@uam.es

Email: jose.garcia_sole@uam.es

\section{J.A. Gonzalo}

Departamento de Física de Materiales, Universidad Autónoma de Madrid, Madrid, 28049, Spain 
and

Escuela Politécnica,

Universidad San Pablo-CEU,

Madrid, 28003, Spain

Email: julio.gonzalo@uam.es

\title{
F. Jaque
}

Departamento de Física de Materiales,

Universidad Autónoma de Madrid,

Madrid, 28049, Spain

Email: francisco.jaque@uam.es

\section{Juan C. del Valle}

Departamento de Química Física Aplicada,

Universidad Autónoma de Madrid,

Madrid, 28049, Spain

Email: juan.valle@uam.es

\section{F. Mallamace}

Dipartimento di Fisica, Università di Messina

and Consiglio Nazionale delle Ricerche - Istituto per i Processi

Chimico-Fisici (CNR-IPCF),

Messina, 98158, Italy

Email: francesco.mallamace@unime.it

\section{H.E. Stanley}

Center for Polymer Studies, and Department of Physics,

Boston University,

Boston, MA 02215, USA

Email: hes@bu.edu

\begin{abstract}
This work reviews several properties of liquid water, including the dielectric constant and the proton-spin lattice relaxation, and draws attention to a bilinear behaviour defining a crossover in the temperature range $50 \pm 10^{\circ} \mathrm{C}$ between two possible states in liquid water. The existence of these two states in liquid water plays an important role in nanometric and biological systems. For example, the optical properties of metallic (gold and silver) nanoparticles dispersed in water, used as nanoprobes, and the emission properties of CdTe quantum dots (QDs), used for fluorescence bioimaging and tumour targeting, show a singular behaviour in this temperature range. In addition, the structural changes in liquid water may be associated with the behaviour of biological macromolecules in aqueous solutions and in particular with protein denaturation.
\end{abstract}


Keywords: water; nanotechnology; biotechnology; crossover temperature; proteins.

Reference to this paper should be made as follows: Maestro, L.M., Marqués, M.I., Camarillo, E., Jaque, D., García Solé, J., Gonzalo, J.A., Jaque, F., del Valle, J.C., Mallamace, F. and Stanley, H.E. (2016) 'On the existence of two states in liquid water: impact on biological and nanoscopic systems', Int. J. Nanotechnol., Vol. 13, Nos. 8/9, pp.667-677.

Biographical notes: Laura Martinez Maestro started collaborating with $\mathrm{PhD}$ before Daniel Jaque obtaining a Bachelor's degree in Physics from the Universidad Autónoma de Madrid, Madrid, Spain, in 2009. Her undergraduate research was focused in confocal microscopy to study the behaviour of laser written waveguides in LiNb. She obtained a Masters degree in Photonics from Universidad Autónoma de Madrid in 2010 and defended her PhD thesis, entitle, "Quantum dots as luminescent nanothermometers: controlled plasmonic hyperthermia" which was awarded with the 'cum laude' remark in the same university five years later. In September 2015, she joined Herz and Johnston groups at the Condensed Matter Physics Department in the University of Oxford as a Postdoctoral Research Assistant to study III-V semiconductor nanowires.

Manuel I. Marqués obtained his BA in Physics at Universidad Complutense de Madrid in 1995 and was awarded with an extraordinary PhD prize in Physics at Universidad Autónoma de Madrid in 2000 under the supervision of Professor Julio A. Gonzalo. He is a Fullbright Fellow at Boston University from 2001 to 2003 where he performed a postdoctoral research in the group of Professor Gene Stanley. In 2003 he was awarded with a Ramón y Cajal appointment at the Universidad Autónoma de Madrid. He is now an Associate Professor in the Material Physics Department. His research interests, mainly focused on phase transitions and light matter interactions, have been awarded with a National Prize of the Cuban Academy of Sciences (2015).

Enrique Camarillo received the BS, MS and a $\mathrm{PhD}$ in Physics from the University National Autonomous of México, UNAM, in 1971, 1985 and 1991, respectively. He was a Postdoctoral Research Fellow in the University Autonomous of Madrid, UAM. Currently, he is working at the Institute of Physics, UNAM, on Optical Properties of Solids.

Daniel Jaque is a Professor at Universidad Autónoma de Madrid. In 2006, he was awarded with the "Young Researcher Award" by the European Association for the Study of Rare Earths and Actanides. That same year, he moved his research interest towards the micro/nano structuration of optical materials by ultrafast laser inscription covering not only practical aspects but also fundamental ones. Indeed, he has been a pioneer in the application of confocal fluorescence imaging techniques for the understanding of the light-matter interaction at the femtosecond time scale. The know-how acquired on confocal fluorescence imaging techniques has allowed him to face new research areas such as fluorescence imaging of living cancer cells and fluids. In 2009, he was an invited Visiting Professor in Heriot Watt University and in Swinburne University of Technology.

José García Solé is a Professor of Applied Physics at Universidad Autónoma de Madrid. He obtained an Invited Professor position at the University of Lyon (France) (1993) and was awarded by a Full Professor position (Cátedra "Elena Aizen de Moshinsky") at the Universidad Nacional Autónoma de México (1994). Recently, he has been also awarded with a special recognition 
by University of Sonora (Hermosillo, México) (November, 2012). His research interest lies on the study of novel optical materials, multifunctional solid state lasers and laser physics. During the last years his research activity has been mostly focused to fluorescence bioimaging and to study new inorganic optical nanocrystals for biomedical applications. He is been co-author of almost 300 peer review papers in international journals and seven books. Among them it does stand out the book "An Introduction to the Optical Spectroscopy of Inorganic Solids” John Wiley \& Sons (2005).

Julio A. Gonzalo has a PhD in Physics (1962) from the Universidad Complutense, Madrid. He did research and teaching at Salamanca (Spain), Mayagüez (Puerto Rico), Rio Piedras (Puerto Rico), Barcelona (Spain) and UAM Madrid (Spain). He worked as Research Collaborator at Brookhaven National Laboratory (US-AEC) and was División Head and Sr. Scientist at PRNC (US-AEC). He has been author or co-author of more than a dozen scientific books, including 'Cosmic Paradoxes' (World Scientific: Singapore, 2012) of which a 2nd edition will appear in 2016.

Francisco Jaque received the $\mathrm{BS}$ and the $\mathrm{PhD}$ in Physics from the Universidad Complutense of Madrid, Madrid, Spain, in 1970 and 1975, respectively. He was a Postdoctoral Research Fellow in the University of Orsay (France), Sussex (UK), Parma (Italy) Strathclyde, Scotland (UK) and Instituto de Física of Mexico (Mexico) working in Radiation Damage and Optical Proprieties of Solids. Since 1982, he is a Full Professor at the Department of Materials Sciences, Universidad Autónoma of Madrid. During the period 2012-2016, he was the University Ombudsman.

Juan Carlos del Valle is an Associate Professor of Applied Physical Chemistry at the University "Autónoma de Madrid". He obtained his PhD in 1994 at the University mentioned above under supervision of Professor Javier Catalán, and did postdoctoral studies at Florida State University collaborating with Professor Michael Kasha. His research focuses on the solvatochromism of organic molecules, the excited state proton transfer reactions of molecular systems possessing intra- and inter-molecular hydrogen bonds and their applications as molecular probes in biological systems or as dye-lasers. His current research includes solvatochromism of molecular probes in water and in water/urea solvent mixtures.

Francesco Mallamace received his BE from the Department of Physics at Messina University in 1973. In 1979, he became Professor of Physics. He started his scientific career at Rome La Sapienza by working on laser experiments related with the theory of coherence of light. After that, he worked on the physics of complex liquids and systems by studying their thermodynamic properties from the stable to the supercooled regime by using several different experimental approaches, such as scattering (light and neutron), viscoelasticity, sound propagation, and nuclear magnetic resonance. In all of these studies, his approach is based on the use of the proper models of statistical physics. His current research interests include water and dynamical properties of glass forming materials (molecular or polymeric) on approaching the arrested-glassy state.

H. Eugene Stanley works in collaboration with students and colleagues attempting to understand puzzles of interdisciplinary science. He has been elected to the U.S. National Academy of Sciences (NAS) and received the 2004 IUPAP Boltzmann Medal. His main current focus understands the anomalous behaviour of liquid water in bulk, nanoconfined, and biological environments. He has also worked on a range of other topics in complex systems, such as 
quantifying correlations among the constituents of the Alzheimer brain and quantifying fluctuations in noncoding and coding DNA sequences, and interbeat intervals of the healthy and diseased heart. His publications, all available online at http://polymer.bu.edu/hes/ have received more than 80,000 ISI Web of Science citations and more than 120,000 citations in Google Scholar. His ISI Hirsch index is currently $h=128$. He has served as thesis advisor to $114 \mathrm{PhD}$ candidates at MIT and Boston University.

\section{Introduction}

The physical and chemical properties of liquid water have been extensively studied for over 100 years using a variety of experimental methods ranging from classical macroscopic techniques, such as the dielectric constant and specific heat, to those that allow additional microscopic information to be obtained, such as X-ray diffraction, Raman spectroscopy, and nuclear magnetic resonance (NMR) spectroscopy. Despite these efforts, the structure of liquid water is still not fully understood. Many models [1-4], have been proposed, including some that had fallen out of use and were then rediscovered, but all of them assume that liquid water is a hydrogen-bonded structure with non-structured features.

It was recently shown that the temperature dependence of the dielectric constant $\varepsilon_{d}$ at $0.1 \mathrm{MPa}$ displays a bilinear behaviour in the $0-100^{\circ} \mathrm{C}$ temperature range, with a crossover temperature $T^{*}$ of approximately $60^{\circ} \mathrm{C}$ [5-9]. Figure 1 shows this behaviour of dielectric constant $\varepsilon_{\mathrm{d}}$ as extracted from four different experimental data in the $0-100^{\circ} \mathrm{C}$ temperature range. These data were obtained over a long period of time. Note that the transition between the two behaviours close to $60^{\circ} \mathrm{C}$ is clear but not well-delimited.

Figure 1 Temperature dependence of the dielectric constant at $0.1 \mathrm{MPa}$ in the temperature range $25-90^{\circ} \mathrm{C}$ (see online version for colours)

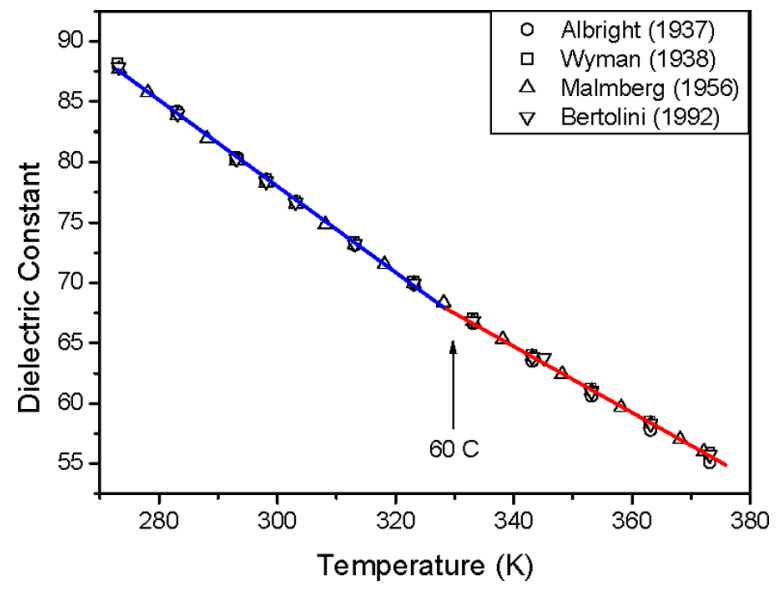

Assuming that a Curie-Weiss law holds for water in this temperature range, the dipole moment of liquid water $\mu$ was determined for both temperature intervals [10]. Thus, we find in the low-temperature range $0-60^{\circ} \mathrm{C}$ a value of $\mu_{1} \approx 2.3 \mathrm{Ds}$, which is close to 
the value for ice. We also calculate, in the temperature interval $60-100^{\circ} \mathrm{C}$, a value of $\mu_{2} \approx 1.8 \mathrm{Ds}$, which is comparable to that reported for the vapour phase [10]. This suggests that there are of two states in bulk liquid water that differ in the amount of their dipole moment. Unfortunately, a correlation between the dipole moment and the microscopic structure of these two states has not yet been determined.

\section{The crossover temperature}

In this work we inspect the existing literature to investigate the temperature dependence of a variety of other physical properties of water, including thermal conductivity (TC) [11], proton spin-lattice relaxation time (T1) [12-14], refractive index (RI) [15], conductivity (C) [16], surface tension (ST) [16-19], and the piezo-optical coefficient (POC) [20]. Figure 2 shows the temperature dependence of all these parameters in the $0-100^{\circ} \mathrm{C}$ interval. With the exception of POCs, we observe a bilinear dependence defining a crossover temperature $T^{*}\left(64 \pm 5^{\circ} \mathrm{C}\right.$ for $\mathrm{TC}, 50 \pm 5^{\circ} \mathrm{C}$ for $T 1$, $50 \pm 5^{\circ} \mathrm{C}$ for RI, $53 \pm 5^{\circ} \mathrm{C}$ for $C$, and $57 \pm 5^{\circ} \mathrm{C}$ for ST). Despite the uncertainty in determining $T^{*}$, these results confirm that in the $0-100^{\circ} \mathrm{C}$ range liquid water presents a crossover temperature in many of its properties close to $50^{\circ} \mathrm{C}$.

Figure 2 Temperature dependence of thermal conductivity, proton spin-lattice relaxation time, refractive index, conductivity, surface tension and piezo-optical coefficient at $0.1 \mathrm{MPa}$ in the temperature range $0-100^{\circ} \mathrm{C}$ (see online version for colours)
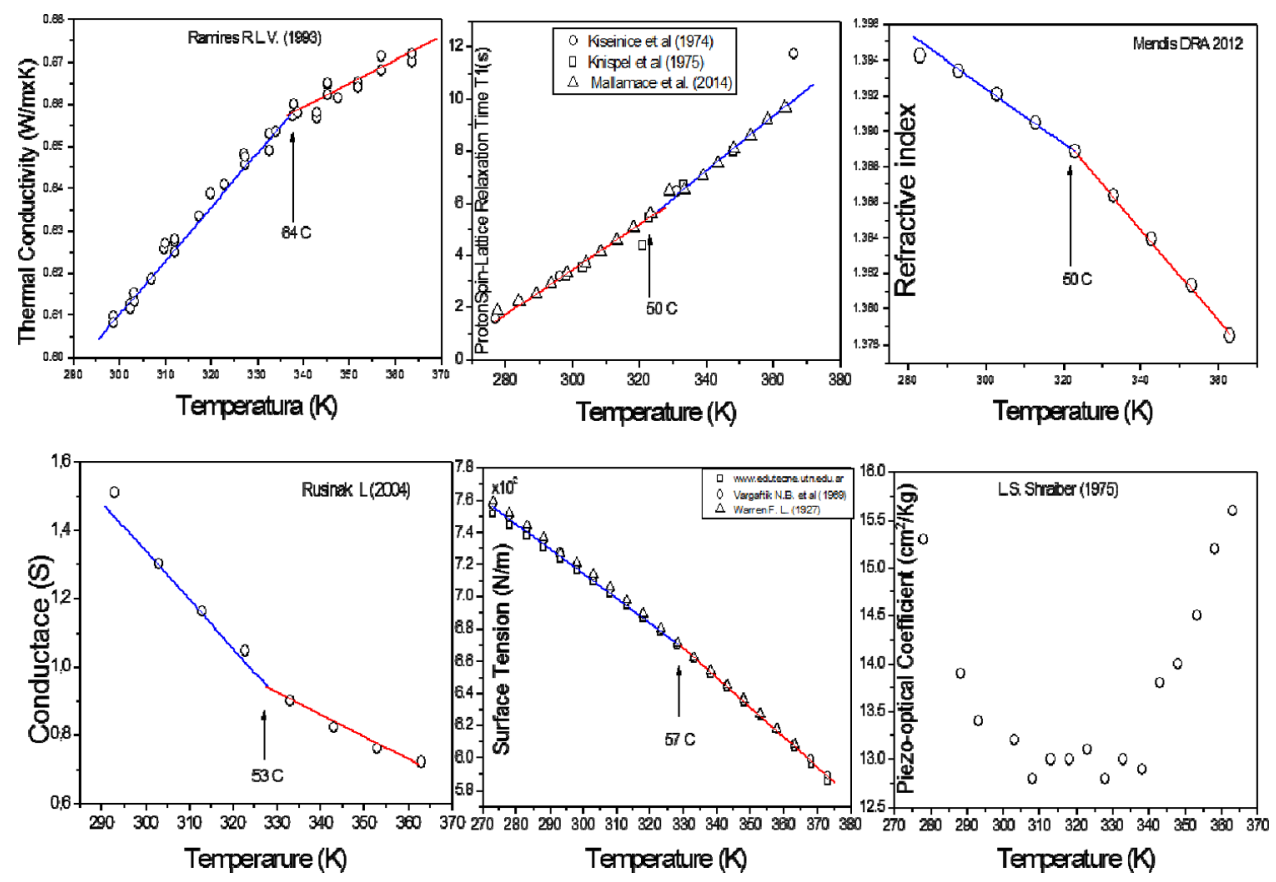

The temperature dependence of $\mathrm{POC}$ in the $0-40^{\circ} \mathrm{C}$ range deviates from the usual behaviour of liquids by showing a downward trend, but above $65^{\circ} \mathrm{C}$ this trend disappears and the behaviour becomes that of typical liquids. 
Other properties of liquid water also show variations in this temperature range. It was recently reported that the thermal expansion coefficient (TEC) presents a crossover temperature at $42 \pm 5^{\circ} \mathrm{C}[14,21]$ and, at the same temperature, the isothermal compressibility shows a minimum [21]. Note that the speed of sound in liquid water, which is correlated with the TEC, also presents a maximum at approximately $60^{\circ} \mathrm{C}$ [22]. These results confirm the existence of a crossover in the behaviour of water that is probably related to the presence of two different liquid states with a temperature shift lying in a broad range between approximately $40^{\circ} \mathrm{C}$ and $60^{\circ} \mathrm{C}$.

\section{Nanoscopic systems}

Regardless the origin of this crossover, it is clear that it plays an important role in nanomedicine, which uses different types of biologically compatible nanoparticles in, e.g., imaging and therapeutics. The biocompatibility of these nanoprobes or nanoagents is based on their stability in physiological fluids, mostly in water. Because this crossover occurs in the $20-60^{\circ} \mathrm{C}$ physiological temperature range, it strongly influences the properties of the nanoparticles currently used for biomedical purpose.

It has recently been found that the optical and structural properties of metallic (gold and silver) metal nanoparticles (MNPs) dispersed in water, which are commonly used as fluorescent nanoprobes and photothermal nanoagents, change significantly above $50-60^{\circ} \mathrm{C}$ [10] owing to nanoparticle aggregation. The increase in MNP size can be measured by the peak wavelength position of the extinction peak associated with their longitudinal surface plasmon resonances. This behaviour has been tentatively ascribed to a subtle structural transformation in liquid water caused by a slight change in the mechanical or electric constants [23].

This result implies that the water transformation may have important implications for other nanoparticles, such as the semiconductor quantum dots (QDs) used in fluorescence bio imaging and tumour targeting. The optical properties of QDs are very sensitive to small changes in their environment, and their characteristic small size and behaviour in surface coatings make them highly stable colloidal nanosensors that can function as probes that signal structural changes in water. In particular, the peak position of QD luminescence is a hypersensitive parameter capable of detecting environment induced changes in the QD size or in the QD-medium coupling.

Figure 3(a) shows the temperature variation of the emission wavelength peak for CdTe QDs (with an average diameter of approximately $1 \mathrm{~nm}$ ) dispersed in water. The insert in Figure 3(a) shows the size distribution of these QDs nanoparticles. The blue points correspond to data recorded during the heating cycle, and the red dots to data recorded during the cooling cycle. Note that

- the emission peak shifts to red as the temperature increases and there is a bilinear dependence with a crossover temperature at approximately $50^{\circ} \mathrm{C}$

- we see the same bilinear relationship in the heating and cooling cycles, which indicates that the CdTe QDs do not aggregate during the thermal cycles, and that this effect must be correlated to the temperature at which the optical measurements are made. 
Figure 3 Temperature dependence of the emission peak wavelength in CdTe QDs. (a) Average QD size: $1.2 \mathrm{~nm}$. (b) Average QD size: $4 \mathrm{~nm}$. The inset in both figures corresponds to the size distribution (see online version for colours)
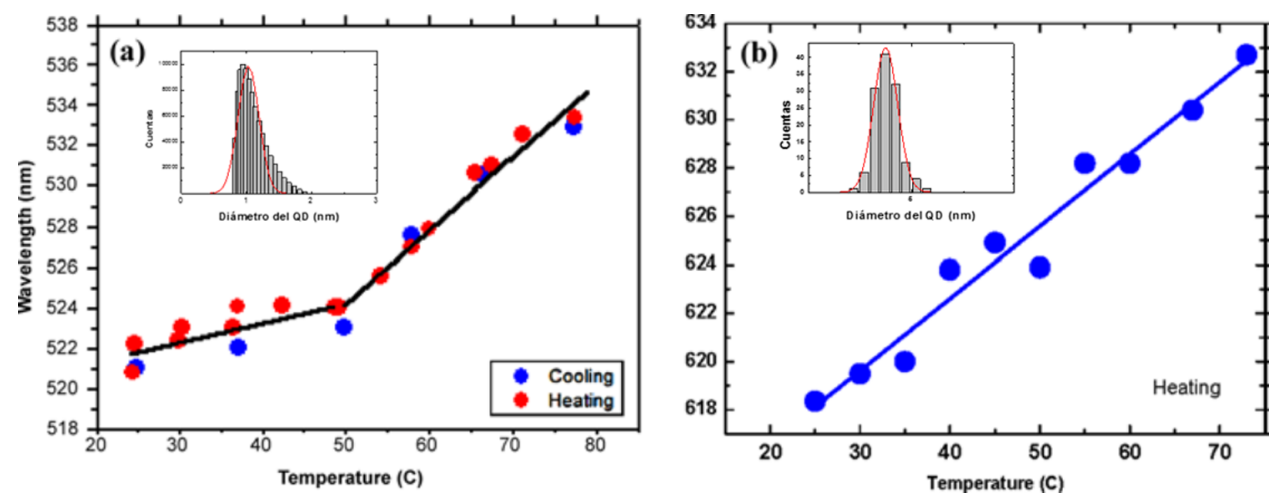

Figure 3(b) shows the temperature variation in the emission wavelength peak but for larger QDs (with an average diameter of approximate $4 \mathrm{~nm}$ ) also dispersed in water. Here the temperature dependence is linear and the bilinear behaviour occurs only in ultra-small QDs.

The bilinear response observed for the ultra-small CdTe QDs has been attributed to an effect related to the coating agent, which modifies the lattice expansion and contraction caused by surface tension [24]. Note that

- when the size of the CdTe QDs is approximately $1 \mathrm{~nm}$, over $90 \%$ of the Cd and Te atoms are located on the surface and most of the excitons responsible for the emission are surface excitons (note also that for $\mathrm{CdSe}$ the Bohr exciton radius is approximately $5 \mathrm{~nm}$ ), and the hyper-sensitivity of these ultra-small dots is related to the liquid characteristics in which they are dispersed

- the approximate crossover temperature $50^{\circ} \mathrm{C}$ is close to the inflexion recently observed for the TEC of bulk liquid water $\left(42 \pm 5^{\circ} \mathrm{C}\right)$ and also to the crossover temperature $T^{*}$ detected in the dielectric constant and in the other properties of liquid water in the $0-100^{\circ} \mathrm{C}$ range mentioned above.

Thus the luminescence of small CdTe QDs nanoparticles could be used as sensors to probe the structure of liquid water as function of temperature in the $0-100^{\circ} \mathrm{C}$ interval.

\section{Biological systems}

This raises the question of whether temperature-driven structural changes in water affect biological macromolecules in aqueous solutions and in particular in proteins, which are the vital functional biological units in living cells. In a recent study by Mallamace et al. [14], the NMR signals of

- bulk liquid water

- water confined on the surface of a protein (lysozyme) were compared. 
It was found that the temperature stability range of the protein is confined to the reversible interval $45-65^{\circ} \mathrm{C}$. In addition, Raman scattering measurements, obtained using multivariate curve resolution (Raman-MCR) have been used to explore the hydrophobic hydration of linear alcohols from methanol to heptanol [25]. The authors conclude that below $60^{\circ} \mathrm{C}$ the hydration shells have a hydrophobic-enhanced water structure with a greater tetrahedral order and fewer weak hydrogen bonds than the surrounding bulk water. This configuration disappears above $60^{\circ} \mathrm{C}$ and is replaced by a structure with weaker bonds. These findings support the existence of two different hydration shells in liquid water with a crossover temperature of $\approx 60^{\circ} \mathrm{C}$.

Figure 4 (a) Temperature dependence of naturation for the protein FKDP. (b) Temperature dependence of denaturation for the protein hole-alpha-lactalbumin. (c) Temperature dependence of the relative value of the hydration shell density for the protein HEW
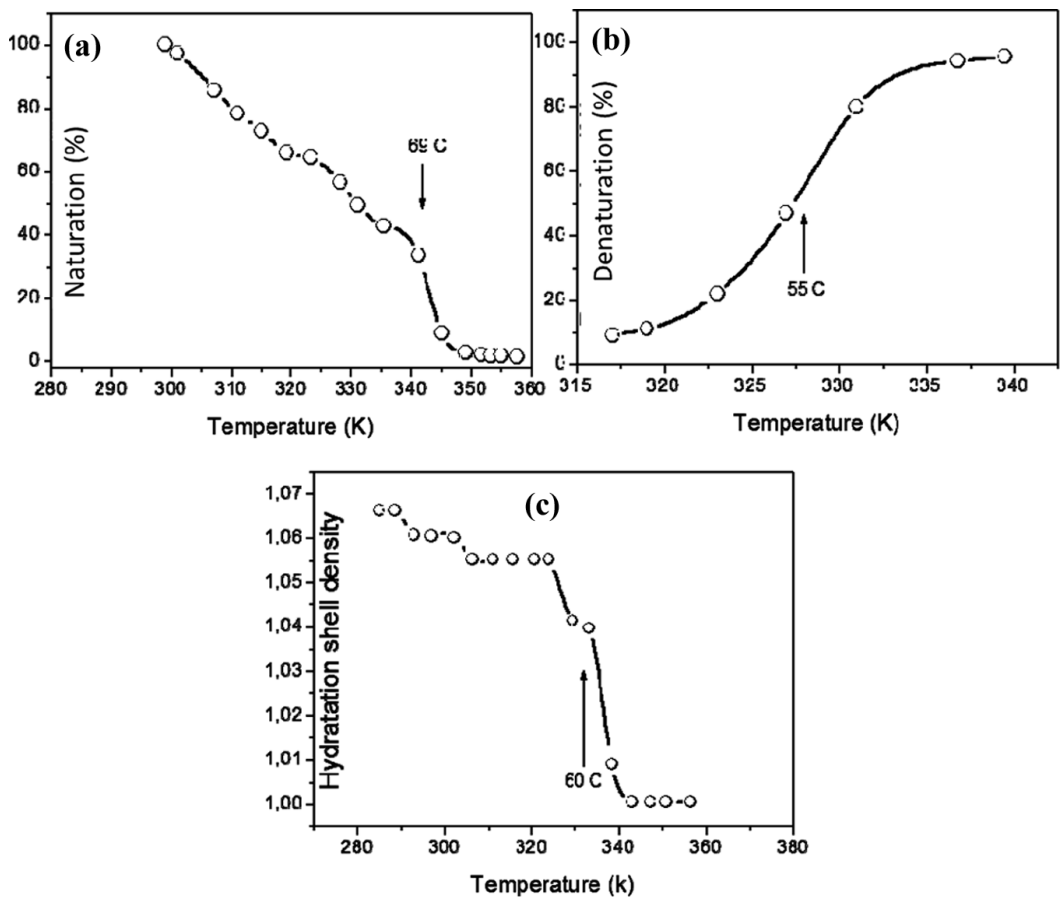

Figure 4 shows the link between the denaturation of proteins and water where the thermal stability of different proteins is measured using different techniques. The data in Figure 4(a) correspond to the stability of the green fluorescent protein (GFP) FKPD-12 measured using the water-bath method [26]. Note that the naturation rate sharply decreases at $\approx 60^{\circ} \mathrm{C}$. A similar result was found using optical methods, although the protein denaturation takes place at a slightly higher temperature. Figure 4(b) shows the denaturation profile for the hole-alpha-lactalbumin protein, as measured by UV spectroscopy in the $32-72^{\circ} \mathrm{C}$ temperature range. The maximum denaturation rate occurs at $\approx 55^{\circ} \mathrm{C}$ and the total denaturation at $67^{\circ} \mathrm{C}$ [27]. Figure $4(\mathrm{c})$ shows the temperaturedependence of the relative hydration shell density (HSD) in the HEWL protein, measured using the wide-angle X-ray scattering (WAXS) technique [28]. Note that the onset and middle-point temperatures are located at $47^{\circ} \mathrm{C}$ and $67^{\circ} \mathrm{C}$, respectively, with the highest 
HSD destruction rate at $\approx 60^{\circ} \mathrm{C}$. In all cases the critical temperature of the protein denaturation is very close to the crossover temperature $T^{*}$ observed in all the properties of liquid water reviewed in this work.

\section{Conclusions}

In conclusion, a review of the physical properties of water in the $0-100^{\circ} \mathrm{C}$ temperature range reveals a bilinear behaviour that defines a crossover temperature at $50 \pm 10^{\circ} \mathrm{C}$. This observation supports the hypothesis that there are two states of liquid water. We find that these two states play an important role in the thermal and optical properties of nanomedical systems. Finally, our preliminary findings suggest that the structure of liquid water strongly influences the thermal stability of proteins. More in-depth research on the thermal stability of proteins dispersed in liquid water is needed.

\section{Acknowledgements}

This work was supported by the Spanish Ministerio de Economía y Competitividad under the following projects, FIS2012-36113-C03, FIS2015-69295-C3-3-P, MAT201347395-C4-1-R and The María de Maeztu Programme for Units of Excellence in R\&D (Grant No. MDM-2014-0377). L.M. Maestro thanks the Secretaría de Estado de Investigación Desarrollo e Innovación for a FPI grant. The Boston University work was supported by DTRA Grant HDTRA1-14-1-0017, by DOE Contract DE-AC0705Id14517, and by NSF Grants CMMI 1125290, PHY 1505000, and CHE-1213217.

\section{References and Notes}

1 Stanley, H.E. (1999) 'Liquid water: a very complex fluid', Proc. 1998 Intl Conf on Complex Fluids, Pramana, Vol. 53, pp.53-83.

2 Plumridge, T.H. and Waigh, R.D. (2002) 'Water structure theory and some implications for drug desing', J. Pharm. Pharmacol., Vol. 54, pp.1155-1179.

3 Riemenschnider, J. (2012) Spectroscopic Investigation on Pure Water and Aqueous Salt in the Mid Infrared Region, Doctoral Thesis, University of Rostok, Germany.

4 Silverstein, K.A.T., Haymet, A.D.J. and Dill, K.A. (2000) 'The strength of hydrogen bonds in liquid water and around nonpolar solutes', J. Am. Chem. Soc., Vol. 112, p.33.

5 Albright, P. (1937) 'Experimental test of recent theories descriptive of the salting-out effect', J. Am. Chem. Soc., Vol. 59, p.2098.

6 Wyman, J. and Ingall, E.N. (1938) 'The dielectric constant of deuterium oxide', J. Am. Chem. Soc., Vol. 60, p.1182.

7 Malmberg, C.G. and Maryott, A.A. (1956) 'Dielectric constant of water from 0 degrees-to 100 degrees', J. Res. Nat. Bur. Stand, Vol. 56, p.1.

8 Bertolini, D., Cassetari, M. and Savetti, G. (1982) 'The dielectric-relaxation time of supercooled water', J. Chem. Phys., Vol. 76, p.3285.

9 Fernandez, D.P., Mulev, Y., Goodwin, A.R.H. and Levelt Sengers, J.M.H. (1995) 'A database for the static dielectric-constant of water and stem', J. Phys. Chem. Ref. Data, Vol. 24, No. 1, p.33. 
10 del Valle, J.C., Camarillo, C., Maestro, L.M., Gonzalo, J.A., Arago, C., Marqués, M.I., Jaque, D., Lifante, G., Solé, J.G., Santacruz-Gomez, K., Carrillo-Torres, R.C. and Jaque, F. (2015) 'Dielectric anomalous response of water at 60 degrees', Philos. Mag., Vol. 85, No. 7 , pp.683-690.

11 Ramires, M.L.V., Nieto de Castro, C.A., Nagasaka, Y., Nagasima, A., Assael, M.J. and Watehan, W.A. (1995) 'Standard reference data for thermal conductivity of water', J. Phys. Chem. Ref. Data, Vol. 24, No. 3, pp.1377-1381.

12 Kiselnik, W., Malyud, N.G. and Toryanic, A.N. (1974) 'Pressure-temperature dependence of nuclear-spin-lattice relaxation in water', J. Struct. Chem., Vol. 15, No. 4, pp.596-598.

13 Knispel, R.R. and Pintar, M.M. (1975) 'Temperature dependence of proton-exchange time in pure water by NMR', Chem. Phys. Lett., Vol. 32, No. 2, pp.238-240.

14 Mallamace, F., Corsaro, C., Mallamace, D., Vasi, S. and Stanley, H.E. (2014) 'Thermodynamic properties of bulk and confined water', J. Chem. Phys., Vol. 141, No. 18, pp.18C504-1, 18C504-10.

15 Mendis, D.R.A. and Samaranayake, W.J.M. (2012) 'Determination of the temperature coefficient of the refractive index of liquids for immersion lithography', Institute of Physics Sri Lanka Proceeding of the Technical Sessions, Vol. 28, pp.77-83.

16 Rusuniak, L. (2004) 'Electric properties of water new experimental data', Acta Geophys. Pol., Vol. 52, pp.63-76.

17 www.eduteone.utn.edu.ar

18 Vargaftik, N.B., Volkov, B.N. and Voljak, L.D. (1983) 'International tables of the surface tension of water', J. Phys. Chem. Ref. Data, Vol. 12, No. 3, pp.817-820.

19 Warren, F.L. (1927) 'XXXIII. The surface-tension balance', Philos. Mag., Vol. 4, No. 21, pp.38-386.

20 Shraiber, L.S. (1975) 'Experimental investigation of thermal dependence of the piezo-optical coefficient of water between 5 and $90^{\circ} \mathrm{C}^{\prime}$, Isr. J. Chem., Vol. 13, pp.181-184.

21 Mallamace, F., Corsaro, C. and Stanley, H.E. (2012) 'A singular thermodynamically consistent temperature at the origin of the anomalous behavior of liquid water', Sci. Rep., Vol. 2, Article No. 993.

22 del Grosso, V.A. and Mader, C.W. (1972) 'Speed of sound in pure water', J. Acoust. Soc. Am., Vol. 52, pp.1442-1446.

23 Maestro, L.M., Camarillo, E., Sánchez-Gil, J.A., Rodríguez-Oliveros, R., Ramiro-Bargueno, J., Caamano, A., Jaque, F., Solé, J.G. and Jaque, D. (2014) 'Gold nanorods for optimized photothermal therapy: the influence of irradiating in the first and second biological windows', RSC Adv., Vol. 4, No. 96, pp.54122-54129.

24 Maestro, L.M., Jacinto, C., Rocha Silva, U., Vetrone, F., Capobianco, J.A., Jaque, D. and Solé, J.G. (2013) 'Response to critical growth temperature of aqueous CdTe quantum dots is non-negligible for their application as nanothermometers', Small, Vol. 9, No. 19, pp.3198-3200.

25 Davis, J.G., Gierszal, K.P., Wing, P. and Ben-Amot, D. (2012) 'Water structural transformation at molecular hydrophobic interfaces', Nature, Vol. 49, pp.582-584.

26 Sagar, D.M., Aoudjane, S., Gaudet, M., Aeppli, G. and Dalby, P.A. (2013) 'Optically induced thermal gradients for proteins characterization in nanolitre-scale samples in microfluids devices', Sci. Rep., Vol. 3, Article number 2130, DOI: 10.1038, srep 02130.

27 Alois, A.R., Lozano, J.M. and Romero, C.M. (2009) 'Holo- $\alpha$ lactalbumin stabilization in the presence of polyols', Rev. Colomb. Quim., Vol. 38, No. 2, pp.209-219.

28 Koizumi, M., Hirai, H., Onai, T., Inoue, K. and Hirai, M. (2007) 'Collapse of the hydration shell of a protein prior to thermal unfolding', J. Appl. Crystallogr., Vol. 40, pp.157-178. 